\title{
On Adaptive Frequency Hopping to Combat Coexistence Interference between Bluetooth and IEEE 802.11b with Practical Resource Constraints
}

\author{
Michael Cho-Hoi Chek and Yu-Kwong Kwok \\ Department of Electrical and Electronic Engineering \\ The University of Hong Kong, Pokfulam Road, Hong Kong \\ Corresponding Author: Yu-Kwong Kwok (email: ykwok@hku.hk)
}

\begin{abstract}
In contrast to traditional frequency hopping techniques, Adaptive Frequency Hopping (AFH) is a low cost and low power solution to avoid interference dynamically. While each AFH algorithm proposed previously is shown to be efficient, a detailed performance analysis of various AFH mechanisms under realistic resource constraints is yet to be done. In particular, based on our performance study on Bluetooth systems presented in this paper, we have found that the AFH mechanism adopted by IEEE 802.15 Task Group 2 (TG2) is very sensitive to memory and power limitations. We then propose a novel Interference Source Oriented Adaptive Frequency Hopping (ISOAFH) approach based on a cross-layer design, in which the baseband layer of Bluetooth considers not only the instantaneous channels condition but also the physical layer transmission characteristics of potential interference sources in determining the hop sequence. In our simulations using detailed MATLAB Simulink modeling, we find that our proposed method is much more robust in that it is insensitive to memory and energy constraints. Indeed, our approach generally achieves a lower collision rate and higher ISM spectrum utilization.

Keywords: adaptive frequency hopping, coexistence mechanisms, Bluetooth, IEEE 802.11b, IEEE 802.15, resource constraints, cross-layer design.
\end{abstract}

\section{INTRODUCTION}

Wireless ad hoc networking using short-range wireless technologies is attractive in that the nowadays ubiquitous hand-held gadgets can communicate when they come close to each other, without the need of pre-installed infrastructure support. In particular, IEEE 802.11x based wireless local area networks (WLANs) systems [2] and Bluetooth based wireless personal area networks (WPANs) systems [1] are the most popular short-range technologies. Both systems operate in the unlicensed $2.4 \mathrm{GHz}$ ISM (Industrial, Scientific, and Medical) frequency band, enabling them to be deployed with a low cost.

However, such a low cost feature comes with a pricedevices using these two different technologies definitely interfere with each other, when they form infrastructureless networks within a close proximity in an ad hoc manner. Indeed, a number of previous studies have shown that this problem could be a severe one [3], [4], [12], [13], [14], [18]. Unfortunately, it is unlikely that we can get rid of any one of these two technologies since they are functionally complementary to each other rather than competing

This research was supported by a grant from the Hong Kong Research Grants Council under project number HKU 7162/03E. ones. Furthermore, both of them are proliferating and expected to operate concurrently in many practical situations. For example, in premises such as shopping malls, convention/exhibition centers, cyber cafes, etc., different people carry devices of both types gather and then possibly interfering each other.

In fact, the physical layer design of Bluetoothfrequency hopping/spread spectrum (FHSS) - can inherently cater for the uncoordinated nature of the $2.4 \mathrm{GHz}$ ISM band [16], while IEEE 802.11b physical layer employs direct sequence spread spectrum (DSSS) scheme. Their interference handling rationales are different in that FHSS tries to avoid interference by hopping among different narrow-band channels whereas DSSS tries to suppress interference by using correlation process. Specifically, the current frequency hopping scheme in Bluetooth is a pseudorandom frequency hopping algorithm. Unfortunately, this pseudo-random mechanism is notoriously sub-optimal. In view of this, the Coexistence Task Group 2 (TG2) [5] of IEEE 802.15 [6] has adopted an adaptive frequency hopping (AFH) mechanism [7], [11] which will be implemented in Bluetooth version 1.2. We elaborate on the details of the $\mathrm{AFH}$ algorithm in the next section.

However, performance analysis of AFH mechanisms under realistic resource constraints are largely ignored by the research community. Specifically, we believe that it is important to study the efficacy of the AFH mechanisms with memory and power constraints, which are very pertinent issues for an optimized implementation in practice. Indeed, despite the advancement and miniature of wireless portable devices, power and memory constraints are still always of prime concern. In this paper, we present our performance analysis of the AFH mechanisms under memory and power constraints, using our detailed modeling based on MATLAB Simulink. We focus on asynchronous connectionless (ACL) links in Bluetooth. Our detailed simulations indicate that the TG2 adopted AFH mechanism is very sensitive to any restrictions in memory size and the channel list updating rate. Effectively, sustaining a satisfactory performance level in the TG2 adopted AFH implies a higher hardware requirement and more computing power (hence, faster battery drainage). In view of this problem, we propose an Interference Source Oriented Adaptive Frequency Hopping (ISOAFH) approach which is more robust in the 
sense that it is insensitive to memory and power limitations. The main idea of our approach is to take into account the radio transmission characteristics of the interference source, and then design a customized channel classification algorithm tightly coupled with judicious AFH control actions such that the two crucial steps - channel classification and adaptive frequency selection - can work together more efficiently. Furthermore, in our study [19], in order to investigate the performance of $\mathrm{AFH}$ in device-crowded area, we have modeled a heterogeneous congested environment such that an IEEE 802.11b and a number of Bluetooth piconets work independently within the same coverage area. For the measurements, our performance analysis is based on some specific metrics for $\mathrm{AFH}$, instead of just based on other aggregate metrics such as goodput.

The rest of the paper is organized as follows. In Section II, we discuss the pseudo-random FH and TG2 adopted AFH mechanisms. Section III presents the proposed ISOAFH mechanism, as well as the simulation platform, models, and scenarios. Section IV describes the simulation results. The last section concludes the paper.

\section{Pseudo-Random Frequency Hopping And Adaptive Frequency Hopping}

\section{A. The Original Pseudo-random Frequency Hopping Scheme}

Bluetooth operates in the $2.4 \mathrm{GHz}$ ISM band from 2402 $\mathrm{MHz}$ to $2843 \mathrm{MHz}$ (in USA and most other countries). The specification defines $791 \mathrm{MHz}$-wide channels for Bluetooth to hop with. The frequency carrier for each packet is determined based on the hop frequency selection scheme that discussed below. In the specification, five different hop sequences are defined which serve for different purposes. In this paper, we will discuss the hop selection scheme for CONNECTION state only.

The original hop selection scheme works as follows. Firstly, a pseudo-random hop sequence will be generated based on 27 bits of master's clock value and 28 bits of the Bluetooth master's address. Secondly, the sequence is mapped to the desired hopping frequency so that each packet is transmitted in a different channel.

Bluetooth is a time division multiplexed (TDM) system with basic unit of $625 \mu \mathrm{s}$ for each time slot. In singleslot packet transmission mode, each packet is carried by different frequency which lasts for a single time slot only. Thus, it results in a nominal hopping rate of 1600 hop/s. In the multi-slot packet transmission modes, which are used only in an ACL connection, packets occupy 3 or 5 time slots without changing its frequency, and therefore, the hopping rate is effectively reduced [1].

A maximum asymmetric data rate $721 \mathrm{kbps}$ could be achieved by using packet type DH5, which is five times larger than DH1. However, it is also more likely to be interfered (or interfering others) since it dwells on a channel five times longer than DH1. Besides, the penalty of losing a DH5 packet is also much higher. This scheme is known as pseudo-random frequency hopping. Although it appears to be random, it is a deterministic process as long as the clock value and master's address remain unchanged. Moreover, it demonstrates some nice properties comparable to the pure random sequence but it is more traceable in real situations [15]. Generally, this scheme performs well with non-static and narrowband interference. However, this is not the case when interferer like IEEE 802.11b WLAN exists nearby, where over $22 \mathrm{MHz}$ of the spectrum is engaged for certain period of time (depends on its loading, data rate, etc.). It is neither non-static nor narrow. Thus, AFH is introduced in order to deal with this prevalent neighborhood.

\section{B. General $A F H$}

AFH is fundamentally different from pseudo-random frequency hopping in that it takes into account the channel condition and changes the hopping frequency dynamically, thereby minimizing interference (both to the $\mathrm{AFH}$ device itself and others) and enabling coexistence with other devices in the $2.4 \mathrm{GHz}$ ISM band.

$\mathrm{AFH}$ is one of the most important non-collaborative coexistence mechanisms. AFH generally consists of two steps: channel classification and adaptive control actions. Specifically, AFH can further divide into 4 sub-problems: (i) device identification; (ii) channel classification; (iii) classification information exchange; (iv) adaptive frequency hopping kernel. Device identification process is mainly used for backward compatibility and checking if a device can work in AFH mode. Channel classification is the process used for distinguishing the channel quality. There are a variety of implementations and this process is very important because the partition sequence generated by AFH kernel is based on the result of this part. Classification information exchange is the protocol for master and slave to exchange the results of their measurement. AFH kernel is the specific algorithm to choose the hop frequency. The goal of the algorithm is to avoid as many bad channels as possible. In this paper, we will focus on the (ii) and (iv) only.

\section{TG2 Adopted AFH Mechanism}

The hopping kernel for TG2 adopted AFH mechanism and the functional blocks inside the AFH module are shown in Figure 1.

Generally, the new AFH mechanism module is placed between the original hop selection kernel and the frequency synthesizer. The new module carries out two functions: sequence generator and remapping functions. The sequence generated specifies when and which frequency to use, and the remapping function is used for maintaining the pseudorandom nature within the partition which behaves as the original hop selection kernel. Firstly, the channels are partitioned two sets, namely "good" and "bad", by some other physical layer mechanisms. A new partition sequence is generated according to the channels' conditions. The newly generated partition sequence is then compared with the original hopping sequence. The adapted hop frequency is generated after the checking of a remapping function. Furthermore, AFH for SCO and ACL connections are treated differently due to their highly different characteristics. In this study, we focus on ACL connection only. 


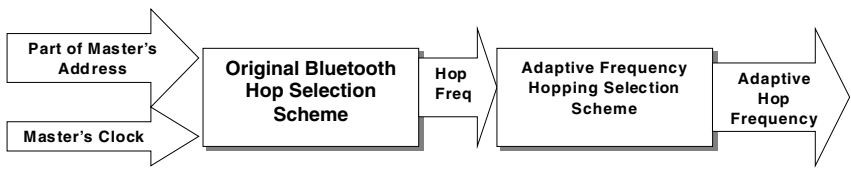

(a) adaptive frequency hopping kernel

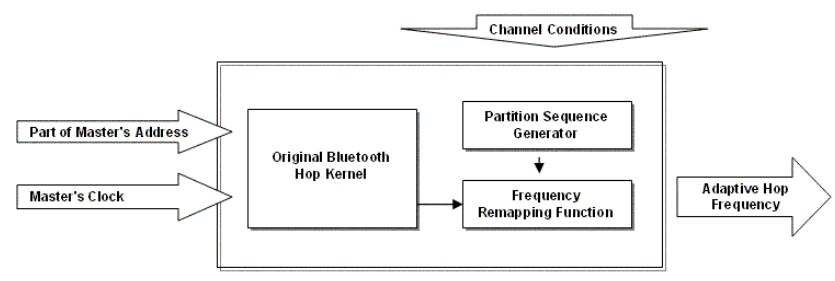

(b) TG2 adopted AFH selection kernel

Fig. 1. Adaptive frequency hopping.

The system running this AFH mechanism examines the channel's quality and maintains a list of "good" and "bad" channels initially. The sequence generator then produces a binary partition sequence which depends on the number of "good" and "bad" channels being available. The system maintains these channel lists and refreshes them periodically. The generated partition sequence is compared to the original hopping sequence, and remapping will take place whenever the two sequences are not referring to the same channel list. The merged AFH mechanism can operate in either Mode $L$ or Mode $H$, depending on $N_{\min }$, the minimum number of channels that $\mathrm{FCC}$ requires Bluetooth to hop over. When the number of available good channels is greater than $N_{m i n}$, the device will operate in Mode $L$ where only good channels are remapped to; otherwise, it will operate in Mode $H$ so that some of the bad channels are still used in order to comply with the FCC regulations. The current $N_{\text {min }}$ allowed in USA is 15 [17]. If "good" channels are less than 15 , then some "bad" channels would still be used which results in a higher chance of packet loss or interference.

\section{Qualitative Analysis of the Existing AFH Mechanism}

After a scrutiny of the AFH mechanism, deficiencies are found in two types of potential environment.

\section{D.1 Environment 1: Resource Limited Environment}

The performance of TG2 adopted AFH mechanism heavily depends on the lists of "good" and "bad" channels, and their validity. It can be further divided into two cases:

1. Memory Concern: In order to make the adopted AFH mechanisms work, the master device must compile a list of "good" and "bad" channels, recording all channels' conditions and their associated information in real-time. Thus, it imposes a great pressure on the memory requirement on hand-held devices. As will be detailed in Section IV, roughly speaking, we find that halving the memory size will double the collision rate.

2. Power Concern: The AFH mechanism heavily depends on the results of channel classification because the formation of the partition sequence depends on the number of "good" or "bad" channels, and the remapping function needs to map "bad" channels to/from "good" channels. Thus, the accuracy of the classification results is crucial for these coexistence schemes. However, the channels' conditions could keep on varying. For example, a channel just sensed to be "good" might turn "bad" right after the classification. As a result, the channel lists need to be updated frequent enough to reflect accurately the current situation.

To be exact, the updating rate of the lists should be faster than or equal to the changing rate of the channels' condition (i.e., the coherence times of the fading processes). This basic requirement imposes stress on the computing power and hence, leads to rapid power drainage, especially in a fast changing environment (e.g., mobility is high).

D.2 Environment 2: Investigation of the Response Time of Channel Estimation

The process of channel estimation is mandatory for all non-collaborative mechanisms; AFH is no exception. However, the estimation process inevitably introduces a response time delay between the presence of interference and the realization of its existence. This response time is one of the most critical parameters for $\mathrm{AFH}$ performance. Although the choice of channel estimation method is vendor implementation specific, some common methods like packet error rate (PER) or bit error rate (BER) are commonly employed. However, these channel estimation methods generally are on a channel-by-channel basis, which requires a longer response time, or induces more errors and higher packet loss. In our simulations, we show that a long response time can lead to more collisions.

\section{The Proposed Approach and Analysis METHODOLOGY}

\section{A. Overview of the Proposed Algorithm}

While pseudo-random frequency hopping hops "blindly" over all the 79 channels, it has been proved to be ineffective in dealing with WLAN system that has wideband and relatively static transmission characteristics. TG2 AFH was then suggested. It outperforms the original pseudo-random frequency hopping because it takes into account the channel quality when choosing a hop frequency. However, we observed that there is still room for improvement with at least two reasons:

- TG2 AFH mechanism does not reveal any consideration of WLAN. In particular, it does not exploit the potential benefits brought by the phenomenon that when one of the Bluetooth channels is subjected to interference from WLAN, there is a high probability that its adjacent channels are also subjected to interference due to the wideband and relatively static radio transmission characteristics of WLAN. 
- Although adaptive control actions are different for all non-collaborative mechanisms, the channel classification methods are more or less the same. With no exception to TG2 AFH, such an approach hinders AFH from achieving higher performance gain.

Thus, in our study, we propose a new approach called Interference Source Oriented AFH (ISOAFH). Our ISOAFH is a further improved mechanism by taking into account the motivations discussed above. In particular, with $802.11 \mathrm{~b}$ radio transmission characteristics considered, we designed ISOAFH with:

1. A customized channel estimation method with faster response time; and

2. A tailor-made adaptive control action that generates hop sequences more efficiently.

\section{B. The ISOAFH Approach \\ B.1 Interference Source Analysis}

We have chosen $802.11 \mathrm{~b}$ as the interference source for illustration purpose. According to the specification of IEEE $802.11 \mathrm{~b}$ [2], the maximum transmitting power is $1000 \mathrm{~mW}$ which spreads on the spectrum with bandwidth of $22 \mathrm{MHz}$. 11 overlapping channels are defined with each separated by $5 \mathrm{MHz}$. However, the only choice for non-overlapping channel configuration is channel 1, 6, 11 (i.e., 2412, 2437, 2462) MHz. With this information known beforehand, we can construct a mapping as shown in Table I to show the frequency usage by both Bluetooth and 802.11b.

TABLE I

FREQUENCY USAGE MAPPING.

\begin{tabular}{l|c|c}
\hline & $802.11 b$ freq. range $(\mathrm{MHz})$ & Bluetooth channels \\
\hline 1 & $2401-2423$ & ch $(0-21)$ \\
2 & $2406-2428$ & ch $(4-26)$ \\
3 & $2411-2433$ & ch $(9-31)$ \\
4 & $2416-2438$ & ch $(14-36)$ \\
5 & $2421-2443$ & ch $(19-41)$ \\
6 & $2426-2448$ & ch $(24-46)$ \\
7 & $2431-2453$ & ch $(29-51)$ \\
8 & $2436-2458$ & ch $(34-56)$ \\
9 & $2441-2463$ & ch $(39-61)$ \\
10 & $2446-2468$ & ch $(44-66)$ \\
11 & $2451-2473$ & ch $(49-71)$ \\
\hline \hline
\end{tabular}

\section{B.2 Customized Channel Estimation}

The objective of our customized channel estimation is to find the carrier of $802.11 \mathrm{~b}$, instead of measuring the channel condition on a channel-by-channel basis. We group the channels into 11 groups according to the channel allocation of $802.11 \mathrm{~b}$ as shown in Table I. The measurement is performed as follows:

ANY of the channels in a group resulted in packet error will be counted as the packet error for that particular group. On the other hand, ALL the groups containing the channel will be counted as well.
With this "overlapping" approach, the reponse time will be much shorter when compared to the ordinary ones.

\section{B.3 Adaptive Control Action}

In ISOAFH, we define 11 modes of revocation, corresponding to the Bluetooth channels affected by $802.11 \mathrm{~b}$ channels. When the results of the customized channel estimation process consider any group of channels as "bad", the master informs slaves to withdraw all the affected channels at once. In this manner, the master does not need to send the messages required by the channel-by-channel methods, and hence, can further shorten the response time.

\section{Analysis Methodology}

Instead of using traditional throughput and/or goodput based analysis which is rather superficial, we try to quantify the performance of various AFH mechanisms by using overall channel collision rate in frequency domain. The overall collision rate diagram shows the rate of occurrence of frequency collision at various levels of interference. Our rationales for using this metric for performance analysis is that the conventional throughput analysis is an aggregate measurement of the whole system performance. As such, it is affected by various parameters in all layers. Thus, it is not a precise performance index for AFH mechanisms. By contrast, the collision rate demonstrates how well or how bad an AFH mechanism works to avoid interference, and it excludes other effects and assesses AFH solely.

\section{Development Tools}

The development platform for the simulation environment is MATLAB and Simulink [8]. MATLAB is the fundamental platform for scientific computing where Simulink is based on. Simulink and a lot of associated toolboxes and blocksets are built on top of the MATLAB, which is used for simulating and modeling a variety of dynamic systems. We have chosen them because it is a widely recognized tool in academic area and it also provides a wide range of specialized libraries for communication system simulations purposes. Moreover, it is highly flexible and extensible that we can develop our own algorithms.

\section{E. Simulation Details and Assumptions}

Figure 2 shows our simulation topology, we model a rife scenario such that various numbers of Bluetooth devices come into proximity and connecting each other with ACL links. Each pair of devices forms a logical connection called piconet which would be able to communicate with packets of type DH1, DH3, and DH5. In addition to the Bluetooth connection, we have also modeled a pair of nodes which forms an IEEE 802.11b independent basic service set (IBSS) network. The number of piconets formed or the traffic loading of the IBSS networks are varied depending on the requirements of individual scenario.

The traffic pattern for Bluetooth connections is modeled as follows. In single-slot mode, masters will keep on sending data to slaves using DH1; slaves will return a NULL packet 


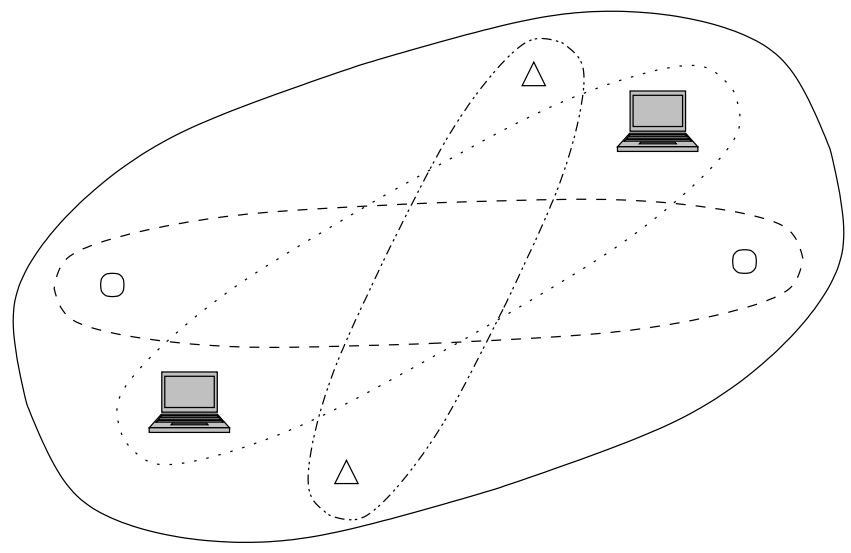

802.11b Connection

$\triangle$-- BT Piconet1

--BT Piconet2

Fig. 2. Simulated environment.

that contains the acknowledgment information. In multislot mode, masters will send data to slaves continuously with DH3 and DH5 packets, which occupy 3 and 5 time slots, respectively. Slaves will return a NULL packet with acknowledgment information which occupies a single slot only.

On the other hand, in multiple piconets environment, each piconet is assigned a fixed master address throughout all the simulations since it will affect the hop sequence (e.g., piconet1 always with address1, piconet2 always with address2, etc.). Moreover, the transmissions of different piconets are modeled with an arbitrary phase delay referenced to a designated piconet, say piconet1.

Measurements are made at the designated piconet1; collision is counted whenever there is overlap in the frequency domain with piconet1's channel. It would only count once in case of multiple interference sources. For multi-slot cases, only multi-slot packets are counted. The general simulation parameters are listed below and in Table II:

- IEEE 802.11b traffic loading step size: 9\%;

- Simulation time: 10 seconds for each interference level in all simulations; and

- Diameter of coverage: $2 \mathrm{~m}$.

TABLE II

Simulation PARAmETERS.

\begin{tabular}{l|l|l}
\hline Parameter & Bluetooth & IEEE 802.11b \\
\hline \hline Load & $100 \%$ & Variable \\
\hline Packet Types & DH1, DH3, DH5 & $11 \mathrm{Mbps}$ \\
\hline Transmitted Power & $1 \mathrm{~mW}$ & $25 \mathrm{~mW}$ \\
\hline \hline
\end{tabular}

\section{Performance Results}

In this section, we describe some results of our simulations. Due to space limitations, we can only present a small set of results here. For the complete set of results, the reader is referred to [19].
We study the effects of memory size and response time of AFH. Figure 3 depicts a comparison of different frequency hopping mechanisms in different transmission mode in DH1, DH3 and DH5, respectively. The fluctuation in the collision rate in DH3 and DH5 might be due to the choice of BT master addresses and the random time offset. Pseudorandom frequency hopping hops "blindly" and its collision rate strictly increases with the WLAN load. For TG2 AFH, due to the limited buffer sizes and relative slow response time, the collision rate still increases with the interference level. On the other hand, ISOAFH keeps a relative lower collision rate in all levels of interference. Another observation is that, in DH3 and DH5, the collision rate does not go up with the WLAN traffic load. This is because interference appears to be more persistent and longer packets are easier to collide. AFH requires less time to response, and hence, results in a lower aggregate collision rate.

\section{Concluding Remarks}

In summary, both the TG2 AFH and Interference Source Oriented AFH (ISOAFH) outperform the original pseudorandom frequency hopping mechanism because extra information about the network environment is considered when choosing a hop frequency. For TG2 AFH, channel lists and refresh rate are the core components of the mechanisms. This mechanism could achieve a very low collision rate when there is no resources constraint. However, its performance could be deteriorating by multiple times when power or memory constraints are present. The proposed ISOAFH, with $802.11 \mathrm{~b}$ specification being considered, customized with both the channel classification and adaptive control processes. It has been proved that such an approach is insensitive to resource constraints. Besides, the simulations indicate that fast and correct estimation of the interference source would achieve a substantial drop in collision rate. Currently, only IEEE 802.11b has been studied due to its popularity. Nevertheless, it is possible to apply similar analysis to deal with other potential interferers such as 802.11g.

\section{REFERENCES}

[1] Bluetooth Special Interest Group, Specifications of the Bluetooth Systems, vol. 1, v.1.0B Core, Dec. 1999.

[2] IEEE Std. 802.11b, "Part 11: Wireless LAN Medium Access Control (MAC) and Physical Layer (PHY) Specifications: Higher-Speed Physical Layer Extension in the 2.4GHz Band," Sept. 1999.

[3] G. Ennis, "Impact of Bluetooth on 802.11 Direct Sequence," IEEE 802.11-98/319, Sept. 1998.

[4] J. Lansford, A. Stephens, and R. Nevo, "Wi-Fi (802.11b) and Bluetooth: Enabling Coexistence," IEEE Network, vol. 15, no. 5, Sept.-Oct. 2001, pp. 20-27.

[5] IEEE 802.15 WPAN Task Group 2 (TG2), http://ieee802.org/15/pub/TG2.html, 2003.

[6] IEEE 802.15.2 Coexistence Mechanisms, http://ieee802.org/15/pub/TG2-Coexistence-Mechanisms.html, 2003.

[7] B. Treister, H. B. Gan, K. C. Chen, H. K. Chen, A. Batra, and O. Eliezer, "Adaptive Frequency Hopping: A Non-Collaborative Coexistence Mechanism," IEEE 802.15-01/252r0, May 2001.

[8] Developers of MATLAB and Simulink for Technical Computing, http://www. mathworks. com/index.shtml, 2003.

[9] C. F. Chiasserini and R. R. Rao, "Coexistence Mechanisms for 


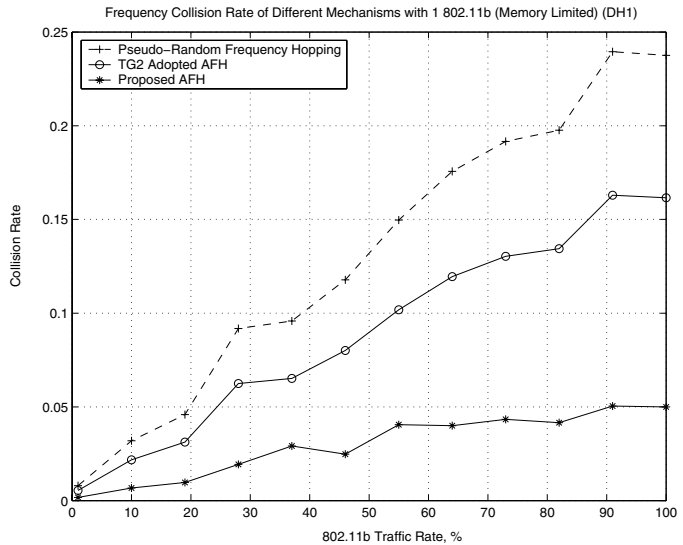

(a) DH1

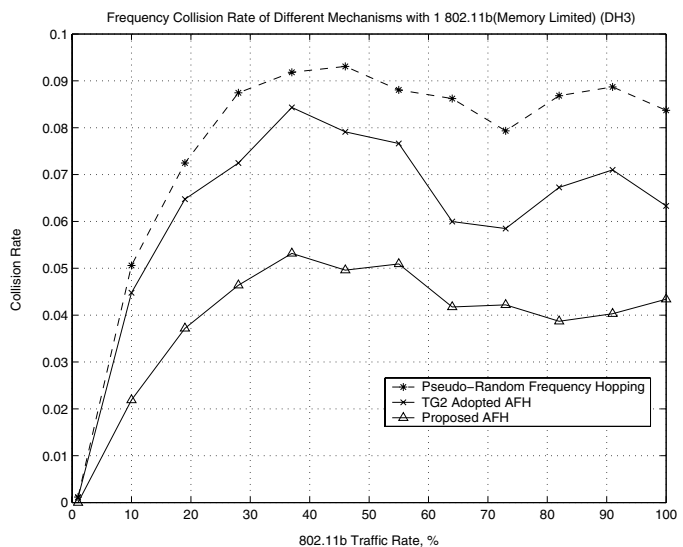

(b) DH3

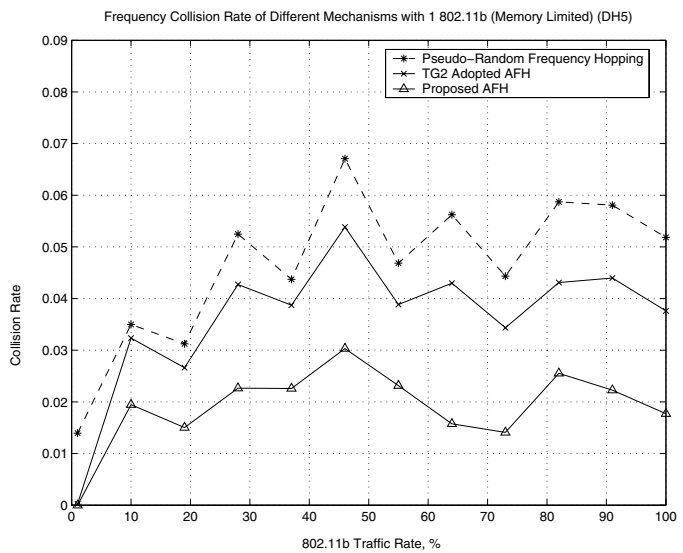

(c) DH5
Interference Mitigation between IEEE 802.11 WLANs and Bluetooth," Proc. INFOCOMM 2002, pp. 590-598, 2002.

[10] N. Golmie, R. E. van Dyck, and A. Soltanian, "Interference of Bluetooth and IEEE 802.11: Simulation Modeling and Performance Evaluation," Proceedings of the 4th ACM International Workshop on Modeling, Analysis and Simulation of Wireless and Mobile Systems, pp. 11-18, July 2001.

[11] Mobilian Technical Papers,

http://www.mobilian.com/.docs/pg/whitepaper.html, 2003

[12] I. Howitt, "WLAN and WPAN Coexistence in UL Band," IEEE Trans. Vehicular Technology, vol. 50, no. 4, July 2001.

[13] C. Cordeiro, S. Abhyankar, R. Toshiwal, and D. P. Agrawal, "A Novel Architecture and Coexistence Method to Provide Global Access to/from Bluetooth WPANs by IEEE 802.11 WLANs," Proc. IEEE Int'l Conference on Performance, Computing, and Communications pp. 23-30, 2003.

[14] J.-L. Chen, H.-S. Wu, and H.-W. Tzeng, "Bluetooth and IEEE 802.11 Coexistence Service Architecture with Fuzzy-based Switching Scheme," NAFIPS 2003, pp. 506-512, 2003.

[15] J. C. Haartsen and S. Zurbes, "Frequency Hop Selection in the Bluetooth Radio System," Proc. 7th Int'l Sym. Spread Spectrum Techniques and Applications, vol. 1, pp. 83-87, 2002.

[16] A. Karnik and A. Kumar, "Performance Analysis of the Bluetooth Physical Layer," Proc. ICPWC 2000, pp. 70-74, 2000.

[17] Silicon Wave, "Data Sheets and Documentation-Coexistence," http://www.siliconwave.com/coexistence.html, 2003.

[18] L. Sydanheimo, M. Keskilammi, and M. Kivikoski, "Performance Issue on the Wireless $2.4 \mathrm{GHz}$ ISM Band in Multisystem Environment," IEEE Trans. Consumer Electronics, vol. 48, no. 3 , pp. 638-643, 2002.

[19] M. C.-H. Chek, Coexistence Techniques for Heterogeneous Mobile Devices Operating in Uncoordinated Radio Spectrum, M.Phil. thesis, The University of Hong Kong, Dec. 2003.

Fig. 3. Collision rate comparison among all mechanisms for different packet types. 